\title{
Welches Verfahren ist zur Diagnostik geeignet?
}

Eine Sarkoidose betrifft bei nahezu allen Patienten (auch) die
Lunge und intrathorakale Lymphknoten. Daher wird bei
klinischem und/oder radiologischem Verdacht auf Sarkoidose
eine Gewebeprobe aus der Lunge und/oder den Lymphknoten entnommen und analysiert. Welche Methode für die Entnahme am besten geeignet ist, habt das Forscherteam um M. B. von Bartheld et al. untersucht. JAMA 2013; 309: 2457-2464

Die endosonografiegesteuerte Feinnadelaspiration aus intrathorakalen Lymphknoten führt zu einer besseren diagnostischen Ausbeute als die klassische flexible Bronchoskopie mit transbronchialer und endobronchialer Lungenbiopsie. $\mathrm{Zu}$ diesem Ergebnis kam die multizentrische, randomisierte Studie mit insgesamt 304 konsekutiv untersuchten Patienten. Bei allen Studienteilnehmern bestand der Verdacht auf eine Sarkoidose Stadium I (mediastinale und/oder hiläre Lymphadenopathie) oder Stadium II (Lymphadenopathie plus Hinweise auf Parenchymbeteiligung).

\section{Endosonografie vs. Bronchoskopie $\nabla$}

Die Patienten wurden randomisiert einem von 2 Verfahren zugeteilt:

- Endosonografie (von Ösophagus oder Bronchien) mit Feinnadelaspiration aus verdächtigen Lymphknoten im Mediastinum oder Hilusbereich $(\mathrm{n}=155)$

- konventionelle Bronchoskopie mit Gewebeentnahme aus Bronchialschleimhaut und Lunge $(n=149)$

Bei allen Patienten erfolgte zusätzlich eine bronchoalveoläre Lavage (BAL). Der primäre Endpunkt war die diagnostische Treffsicherheit der jeweiligen Methode zum Nachweis der klassischen, nicht verkäsenden Granulome, bezogen auf die endgültige Diagnose 6 Monate nach der Untersuchung. Sekundäre Endpunkte umfassten u.a. unerwünschte Ereignisse bei der Untersuchung.

\section{Mehr charakteristische Granulome gefunden $\nabla$}

Bei der Auswertung zeigte sich, dass nach der endosonografisch gesteuerten Aspiration deutlich mehr charakteristische Granulome gefunden worden waren als nach einer Bronchoskopie mit Biopsie (114 vs. 72 Patienten; $p<0,001)$. Die diagnostische Ausbeute (Anzahl von Patienten mit Granulomnachweis dividiert durch die Gesamtzahl der Patienten mit der endgültigen Diagnose einer Sarkoidose) betrug 80\% für die Endosonografie und 53\% für die Bronchoskopie.

In der Bronchoskopiegruppe traten 2 schwere Komplikationen auf: ein Pneumothorax, der mit einer Thoraxdrainage versorgt werde musste, und eine respiratorische Insuffizienz, die die zeitweilige nicht-invasive respiratorische Unterstützung notwendig machte. In der Endosonografiegruppe kam es bei einem Patienten $\mathrm{zu}$ einem Mediastinalabszess, der über eine Thorakotomie ausgeräumt und anschließend längere Zeit antibiotisch behandelt werden musste.

\section{Fazit}

Bei Verdacht auf eine Lungensarkoidose Stadium I oder II kann die endosonografisch gesteuerte Aspiration aus einem Lymphknoten die Diagnose sichern und schneidet dabei besser ab als die flexible Bronchoskopie mit Gewebeentnahme, so die Autoren.

\section{Dr. Elke Ruchalla, Trossingen}

\section{Neuer Therapieansatz beim IPEX-Syndrom}

Das IPEX-Syndrom ist eine seltene Erkrankung, von der vor allem Neugeborene und Kleinkinder betroffen sind. Die jungen Patienten leiden - oftmals gleichzeitig - unter schwerem Durchfall, Diabetes, Hautentzündungen, Blutarmut, Lungenentzündung und Hepatitis. Ursache ist ein Defekt in der Immunabwehr. Einem Team um Prof. Falk Nimmerjahn vom Lehrstuhl für Genetik der Friedrich-Alexander-Universität Erlangen-Nürnberg (FAU) ist es nun gelungen, einen neuen Therapieansatz für diese Autoimmunerkrankung zu identifizieren. Die Ergebnisse ihrer Arbeit haben sie im November 2013 im Fachjournal „Proceedings of the National Academy of Sciences“ (PNAS) publiziert.

Betroffen von der Erkrankung sind Kinder, denen durch einen Gendefekt ein Eiweißstoff fehlt, den der Organismus braucht, um regulatorische T-Helferzellen zu produzieren. Diese Zellen sorgen dafür, dass das Immunsystem nicht das körpereigene Gewebe angreift. Fehlen die Zellen, sind schwere Entzündungen die Folge, die Gewebe im gesamten Organismus zerstören können. Unbehandelt endet die Krankheit oft tödlich. Eine der wenigen Therapien, mit denen sich das IPEX-Syndrom heilen lässt, ist eine Knochenmarktransplantation. Diese ist jedoch für die geschwächten Patienten sehr belastend.

Dem Team um Nimmerjahn ist es nun gelungen, einen Krankheitsmechanismus zu identifizieren, der zu Entzündungen von Leber, Niere, Haut und Lunge beiträgt. Es sind die Autoantikörper, die in den B-Zellen gebildet werden und die für die Gewebeentzündungen verantwortlich sind. Als die Wissenschaftler die B-Zellen medikamentös aus dem Organismus entfernten, wurden auch die Entzündungen schwächer und die Überlebensrate stieg deutlich an. Da diese Therapie bereits bei einer Vielzahl anderer Autoimmunerkrankungen eingesetzt wird, hoffen die Forscher nun, dass sie auch beim IPEX-Syndrom rasch in die klinische Praxis übertragen werden kann.

Nach einer Mitteilung der FriedrichAlexander-Universität Erlangen-Nürnberg 